\title{
TABLE GÉNÉRALE
}

DES MATIËRES

CONTENUES DANS LES

\section{VINGT PREMIERS VOLUMES}

DU

\section{BULLETIN INTERNATTONAL}

DES

\section{SOCIETES DE LA CROIX-ROUGE}

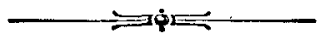

(ANNÉES 1869 A 1889) 


\section{TABLE GÉNÉRALE DES MATIĖRES}

CONTENUES DANS LES VINGT PREMIERS VOLUMES DU BULLETIN INTERNATIONAL DES SOGIÉTÉS DE LA CROIX-ROUGE

(Annèes 1869 à 1889)

\section{COMTTÝ INTERNATIONAL}

Personnel et affaires intérieures

Personnel, XV, 5, 121; XIX, 55 (69e Circ.)

Le Dr Maunoir (nécrologie), I, 7, 61.

Le général Dufour (nécrologie), IV, 107.

Le colonel Edmond Favre (nécrologie), XI, 97.

Louis Micheli-de la Rive (nécrologie), XIX, 56.

Têtes de lettres, I, 6 (17e Circ.)

Bibliothèque, ]X, 197.

Ouvrages reçus (dans tous les Bulletins, depuis II, 194).

Participation à l'exposition de Vienne (1873), V, 7 .

Participation à l'exposition de Paris (1878), IX, 200.

\section{Travaux divers}

Conférence des Soc. de la Croix-Rouge à Genève (1864), XV, 126.

Guerre franco-allemande (1870-71), II, 3, 77, 92 (24e Circ.), 165; III, 113; IV, 1 .

Projet de conférence internationale à Genève (1871), 11, 180 (26 Civc.); III, 1 (2\% Circ.)

Projet de conférence internationale à Vienne, $\mathrm{X}, 65$. 
Ambassade japonaise à Genève (1873), V, 11.

Affaire du Virginius (1873), V, 81.

Conférence de Bruxelles sur les lois de la guerre (1874), V, 197 (30 Circ.); VI, 9.

Insurrection de l'Herzégovine (1875), VI, 175; VII, 1.

Mission au Montenegro (1876), VII, 55.

Guerre turco-russe (1877-78), VIII, 143 (37e Girc.), 147 (38 Girc.), 170) (39e Circ.); IX, 32, 115, 273 (44e Circ.)

Concours sur l'art d'improviser des moyens de secours (1881):

Programme, XIlI, 5 (47e Circ)

Résultats, XIV, 55, 91, 141 (51e Circ.); XV, 5.

Troisième conférence internat. des Soc. de la Croix-Rouge à Genève (1884) :

Consultation des Comités centraux, XV, 6, (52e Circ.)

Convocation et préparatifs, XV, 51 (53e Circ.), 53 (54e Circ.), $122\left(55^{\mathrm{e}}\right.$ Circ.), 182 (56e Circ.)

Historique, par M. Ed. Odier, XV, 185.

Résolutions et vœux, XV, 198.

Conférence du Dr Port et exposition de moyens de secours improvisés, $\mathrm{XV}, 208$.

Compte rendu, XVI, 55, 97.

Concours d'Anvers pour baraque d'ambulance transportable (1885):

Annonce, XVI, 42 (57e Circ.)

Programme, XVI, 44.

Renseignements divers, XVI, 56, 97.

Résultats : Rapport du jury, XVI, 129 (59e Circ.)

Guerre serbo-bulgare (1885-1886, XVlI, 5, 14 (63e Civc), 80.

Rôle du Comité international et relations des Comités centraux entre eux (1885-1887):

Enquête, XVI, 53 (58 Circ.), 98; XVIII, 97 (66 Circ.)

Conclusions, XVIII, 99.

Monument de la Croix-Rouge (1885-87):

Projet de M. Kissling (1882, XuI, 156.

Etude, XVI, 98.

Rapport de M. C. FAvre, XVIII, 101.

Conférence internationale de Carlsruhe (1887), XVII, 185.

La Croix-Rouge sur mer (1887), XIX, 87 (71c Circ.)

Vingt-cinquième anniversaire de la fondation de la Croix-Rouge (1888):

Le 9 février, XIX, 61.

Le 26 octobre, XIX, $58(70 \mathrm{e} \mathrm{Circ.}), 92 ; \mathrm{XX}, 1$.

Mémorial et tableau, XIX, 149.

Emploi abusif du signe et du nom de la Croix-Rouge (1888) :

Exemples, V, 9 ; VI, 13; XI, 4; XIX, 38 
Enquête, XIX, 141 (72e Circ.)

Rapport de M. A. GaUtier, XIX, 143; XX, 26.

Concours, XX, 27 (73e Circ.), 141, 177.

\section{Circulaires concernant diverses Sociétés nationales}

Exposition de La Haye (18e Girc.), I, 7.

Appel aux neutres pour la guerre civile espagnole (29e Circ.), V, 145, 194, 242 ; VI, 173.

Formation de la Soc. monténégrine (31E Circ.), VII, 66.

Formation de la Soc. serbe (32e Circ.), VII, 118.

Appel aux neutres pour la guerre de Serbie (33e Circ.), VII, 120.

Formation de la Soc. roumaine (34e Circ.), VII, 159.

Dissolution de la Soc américaine (34e Circ.), VII, 159.

Formation d'une nouvelle Soc. ottomane ( $36^{\circ}$ Circ.), vill, 39.

Formation de la Soc. grecque (40e Girc.), vIII, 176.

Avis relatif à la Soc. belge (42e Circ.), IX, 8.

Appel en faveur de la Soc. serbe (42e Circ.), IX, 8.

Appel en faveur de la Soc. grecque (43e Circ), IX, 113.

Formation de la Soc péruvienne (45e Circ.), XI, 98.

Appel en faveur de la Soc. péruvienne (46 Circ.), XII, 5.

Formation de la Soc. argentine (48e Circ.), XIII, 10.

Formation de la Soc. hongroise ( $49 \mathrm{e}$ Circ.), XIII, 13.

Reconstitution de la Soc. américaine (50 e Circ.), XIII, 185.

Formation de la Soc. bulgare (60e Circ.), XVI, 137.

Appel en faveur de la Soc. bulgare (61e Circ.), XVII, 11.

Appel en faveur de la Soc. serbe (62e Circ.), XVII, 12.

Remerciements de la Soc. bulgare $\left(64^{\circ}\right.$ Circ.), XVII, 73.

Communications de la Soc. italienne (65e Circ.), XVII, 74.

Reconstitution de la Soc. portugaise (67e Circ.), XVIII, 153.

Formation de la Soc. japonaise ( $68 \mathrm{e}$ Circ.), XVIII, 157.

Enquête provoquée par la Soc. hongroise (720 Circ.), XIX, 141.

Formation de la Soc. congolaise et africaine (75e Circ.), XX, 76.

\section{Variétés}

Enquète sur les législations militaires, I, 62, 108, 162.

Ce que c'est que la Croix-Rouge, par G. Moynier, VI, 1.

La Croix-Rouge usurpée, V, 9 ; VI, 13 ; XI, 4.

Du double caractère, national et international, des Soc. de la Croix-Rouge, I, 159.

De l'adoption d'une dénomination uniforme, Ill, 177. 
Les Soc. de la Croix-Rouge pendant la guerre franco-allemande, II, 1.

Des bureaux de renseignements pendant la guerre franco-allemande, $\mathrm{V}, 148$.

Les dix premières annėes de la Croix-Rouge, par G. Mornier, IV, 165.

Les Soc. de la Croix-Rouge et la guerre d'Orient, VIII, 92.

Les journaux de l'oeuvre, J, 59, 119, 163.

Nëcrologe général de la Croix-Rouge, IV, 138.

L'avenir de la Croix-Rouge, par G Moynier, XIII, 65

La chronologie de la Croix-Rouge, IV, 137; IX, 200 ; XIII, 113.

Décisions prises dans les conférences internationales, $\mathrm{XX}, 61$ (7/c Circ.)

Une lecture sur la Convention de Genève à l'Institut de France, l, 8.

Ratification des articles additionnels à la Convention de Genève, I, 6, 107, 159 ; II, 96 ; VI, $112 ;$ XIV, 59.

La Convention de Genève pendant la guerre franco-allemande, par G. MovNIER, IV, 51, $104 ;$ II, 10.

De la revision de la Convention de Genève, VIl, 122.

La Convention de Genève pendant la guerre turco-serbe, VII, 164 (35e Circ)

La Convention de Genève au Japon, VIII, 1.

La Convention de Genève pendant la guerre turco-russe, VIII, 41, 83, 154; IX, 11, 119.

La Convention de Genève en Bosnie, $\mathrm{X}, 1$.

Le shah de Perse et la Convention de Genève, V, 16.

Des formules d'adhésion à la Convention de Genève, par G. Moynier, $\mathrm{XX}, 80$.

\section{Publications}

Bulletin :

Avant-propos, I, 1.

Prospectus, J, 4 (16 Circ.), 59.

Aux abonnés, V, 193.

Les huit premières années du Bulletin, IX, I (41 Circ.)

Le passé et l'avenir du Bulletin, XI, 1.

Actes du Comité international, II, 186.

Note sur une instítution judiciaire internationale, par G. MoYNIEk, III, 121. (28e Circ)

Edition française du livre de Lueder sur la Convention de Genève, VII, 115. Compte rendu de la Conférence internationale d: Genève (1884), XVI, 55, 97. Les circulaires du Comité international, XVII, 75.

Mémorial des vingt-cinq premières annéeg de la Croix-Kouge, XIX, 149. 


\section{EUROPE}

\section{ALLEMAGNE \\ Croix-Rouge allemande}

Organisation générale, I, 14, 64; XVII, 209; XVIII, 81.

Comité central des Associations allemandes de la Croix-Rouge :

Présidence, III, 186.

Activité générale, IV, 70, 139. (Voir, pour la suite, la Correspondance de Berlin, que contient chaque Bulletin, sauf ceux d'octobre 1887 et d'octobre 1889.)

Guerre franco-allemande, Il, I1, 114, 202; III, 8; IV, 6, 73.

Instruction sur la Convention de Genève, IV, 74.

Rapport décennal, XI, 151.

Concours divers:

a) Manuel de chirurgie, V, 18; VI, 25.

b) Etude sur la Convention de Genève, V, 18; VI, 26, 125, 172.

c) Aménagement intérieur des baraques d'ambulance: Jer Concours: Annonce, XIX, 10.

Programme, XIX, 11.

Résultat négatif, XIX, 155.

2me Concours : Annonce et programme, XX, 34.

Rapport du jury, XX, 144.

Le Kriegerheil, I, 66.

Guerre serbo-bulgare, XVII, 89, 191.

Conférence internationale de Carlsruhe (1887):

Annonce et préparatifs, XVII, 185; XVIII, 6, 111, 114, 115.

Programme, XVIII, 116.

Protocoles, XIX, 9.

Confẻrence allemande de Nuremberg (1870), I, 170; III, 57, 60. Assemblée de Göttingen (1883), XV, 63 .

Les Soc. allemandes pendant la guerre turco-russe, IX, 221.

Union des Soc. de femmes allemandes, III, 13; Vl, 27.

Les Soc. de la Croix-Rouge et les Soc. de temmes, VIII, 104.

Soc. patriotique de femmes, I, 68, 168; IV, 142; XV, 64. 


\section{Variétés}

Ordonnance du 28 mai 1873 (sur les secours volontaires), V, 89.

$\begin{array}{llll}" 10 \text { janvier } 1878 & \# & \text { " } & \text { IX, } 209 . \\ 3 \text { sept. } 1887 & " & n & \text { XIX, } 17 .\end{array}$

》 4 janvier 1883 (sur le costume des volontaires), XIV, 96.

Statistique du personnel sanitaire pendant la guerre franco-allemande, V, 23.

Les pansements antiseptiques dans l'armée allemande, XVII, 20.

Kaiser-Wilhelm-Stifiung (pour les invalides), IV, 143.

Fondation nationale Victoria (pour les invalides), XVI, 64.

Association des infirmiers volontaires, XVIII, $201 ; \mathrm{XX}, 57$.

Euvre des Samaritains, XIII, 90; XIV, 32, 175; XVI, 145; XVII, 250, 281.

Exposition allemande d'hygiène et de sauvetage à Berlin (1883); XIV, 168 ; XV, 45.

Exposition de matériel d'ambulance à Berlin, XX, 150 .

\section{Bibliographie}

Violations de la Convention de Genève par les Français, II, 206.

Jes sépultures des soldats allemands en Belgique, par le Dr HovER, XIII, 89. Voyage d'hiver de Berlin au théâtre de la guerre serbo-bulgare, par O. DE HCENIKA, XVII, 89.

Rapport sanitaire sur les armées allemandes pendant la guerre contre la France en 1870-1871, XVI, 9; X VIII, 47.

La confẻrence de Carlsruhe, par DE KNesebeck, XIX, 96.

\section{Prusse}

\section{Croix-Rouge prussienne}

Personnel et activité générale, I, 11; IV, 29, 12 .

M. R. de Sydow (nécrologie), III, 137.

Le Dr Löffler (nécrologie), v, 177.

Bernhardt von Langenbeck (necrologie), XIX, 15.

Compte rendu de la Conférence internationale de Berlin, I, 18.

Vingt-cinquième anniversaire de la fondation, $\mathrm{XX}, 120$.

Concours sur la Croix-Rouge dans les giıerres navales, II, 121.

Concours sur l'hospitalisation près des champs de bataille, XX, 128.

Société d'Altona, I, 19; III, 105, 143; VIl, 104; XII, 147; XVI, 82.

d d'Anhalt, III, 108.

" de Francfort, III, 225; IV, 157.

"de Hanovre, I, 120; VI, $52 ;$ XVIII, 24.

"de Kiel, IV, 29.

n patriotique des dames, XX, 208. 


\section{Variètés}

Instruction pour le service sanitaire de l'armée, I, 165.

Signal pour les navires en détresse, I, 18.

Hygiène militaire, I, 72.

Hôpital Augusta, à Berlin, I, 122.

Blessures par projectiles de plomb mou, V, 240.

Effets des balles de fusils modernes, XVI, 151.

Neutralisation des stations balnéaires, XVI, 120.

\section{Bibliographie}

Revue d'hygiène publique, 1, 19 .

Hôpitaux et baraquements à Berlin, par SternberG, III, 38.

Baraques-lazarets à Aix-la-Chapelle, par AdEnaw et von KAven, IV, 31 .

Etude sur les Sociétés de secours, par Friedleben, IV, 32.

Hôpitaux et infirmières, d'après NIESE, V, 68.

Blessés militaires avant la Croix-Rouge, par Gurtt, V, $129 ; \mathrm{X}, 78$.

Assistance privée en temps de guerre, par ОснWADt, VI, 94.

La Convention de Genève et les Soc. de secours, d'après Schmidt-ERnst-

HAUSEN, par E. ODIER, VI, 153, 203; VII, 37.

Chirurgie de guerre, par le Dr Esmarch, X, 27.

Traitement antiseptique, par le Dr Esmarch, X, 128.

I) l'humanité dans la guerre, par le Dr Lueder, XI, 174.

Manuel des Soc. de dames de la Croix-Rouge, XII, 148.

La Croix-Rouge et le droit des gens, par le Dr Treuenpreuss, XII, 237.

Les transports sanitaires par chemins de fer, par J. zUR NIEDEN, XII, 149;

XIII, 167.

L'improvisation des moyens de secours, d'après le Dr Rase, XVI, 80.

Une brochure sur l'histoire de la Croix-Rouge, par le Dr Treuenpreuss, XVIII, 200.

Des baraques sanitaires portatives, par les Drs DE JAANGENBECK, DE CoLER et Werner, XVII, 266.

Utilité des femmes en temps de guerre, par le Dr Esmarch, XVIII, 117.

\section{Pays se rattachant à l'organisation prusaienne}

A) SAXE-ALTENBOURG

Guerre franco-allemande, III, 107.

$$
\text { B) ANHALT }
$$

Guerre franco-allemande, III. 108 , 


\section{C) HAMBOURG}

Comitẻs réunis, V, 68.

G. von Lind (nécrologie), XII, 140.

Soc. des dames, XVI, 20.

\section{D) MECKLEMBOURG-SGHWERIN}

Activité générale en :

$\begin{array}{llll}1870-71 & \text { III, } 109 & 1879-80 & \text { XII, } 142 . \\ 1871-72 & \text { V, 67. } & 1880-81 & \text { XIII, 158. } \\ 1872-73 & \text { V, 239. } & 1881-82 & \text { XIV, 161. } \\ 1873-74 & \text { VI, 94. } & 1882-83 & \text { XV, 247. } \\ 1874-75 & \text { VII, 149. } & 1883-84 & \text { XVI, 180. } \\ 1875-76 & \text { VIII, 130. } & 1884-85 & \text { XVII, 247. } \\ 1876-77 & \text { IX, 255. } & 1885-86 & \text { XVIII, 199. } \\ 1878-79 & \text { XI, 171. } & 1886-88 & \text { XX, 56. }\end{array}$

E) OLDENBourg

Activité générale en 1871 IV, 93

$1872 \mathrm{~V}, 66$.

1877 XI, 11.

Bade

Croix-Rouge badoise

Activilé générale :

A) Soc. des Dames en 1872 V, 31. 1880 XII, 197.

1874 VI, $182 . \quad 1882$ XIV, 147.

1875 VII, $134 . \quad 1883 \mathrm{XV}, 217$.

$1878 \mathrm{X}, 113$.

1879 XI, 153.

b) Soc. des Hommes 1874-77 IX, 225. 1882-85 XVII, 282.

C) Association générale 1879 V, 30 1878-81 XIII, 204.

1873 V, 221. 1882-86 XVIII, 173.

1876-77 IX, 288.

Guerre franco-allemande, II, 28; III, 61, 190.

Suppression des "Nachrichten ", III, 143.

Réunion de Carlsruhe en 1874, Vl, 183.

Formation et emploi des infirmières, I, 128.

Corps des brancardiers volontaires, V, 217 ; XIV, 11 ; XVI, 152.

Asile de convalescents à Bade, II, 126.

Le Dr Steiner (nécrologie), IV, 146.

Conférence internationale de Carlsruhe en 1887 (voy. Allemagne). 


\section{Variétés}

La fabrique Lipowski, à Heidelberg, III, 195.

\section{Bibliographie}

Le premier secours, par Pezet de Conval, I, 21.

La Convention de Genève pendant la guerre de 1870-71, par v. C., III, 15, 187. Observations sur la Convention de Genève, par BLunTschl, IV, 76.

Catalogue de la bibliothèque des Soc badoises, Vl, 32.

Histoire de la Soc. badoise des dames, .XIII, 36.

\section{Bavière}

\section{Croix-Rouge bavaroise}

Activité générale en 1872 et 1873 VI, $79 . \quad 1882$ et 1883 XVI, 11 .

1874 à 1870 VIIJ, 184. 1880 à 1884 XVII, 31 .

1877 à 1879 XI, $153 . \quad 1886$ et 1887 XIX, 22.

Guerre franco-allemande, II, 33 ; UI, 63 ; IV, 78.

Protectorat de la Soc., XVIII, 9.

Société des Dames :

Formation, I, 134.

Activité générale de 1881 à 1883, XV, 219.

\section{Bibliographie}

Transport des blessés, par VogL et Schmidt, V, 31.

La Convention de Genève, par Lueder, VII, 79

Edition française du même ouvrage, VII. 115.

Les conférences internationales de la Croix-Rouge, XIV, 13.

L'improvisation des moyens de secours, d'après le Dr PoRT, par le Dr APPIA, $\mathrm{XV}, 66$.

\section{Hesse}

Croix-Rouge hessoise

Programme en temps de paix, I, 91 .

Guerre franco-allemande, II, 45 ; IV, $25,89$.

Assistance des invalides, $\mathrm{V}, 62$.

Activité générale de 1871 à 1874, V, 171 ; VI, 43.

Guerre turco-russe, IX, 304.

\section{Variétés}

Corps de gymnastes, l, 137. 


\section{Bibliographie}

Publications de la Socièté hessoise, I, 29.

\section{Saxe}

Croix-Rouge saxonne

Personnel du Comité, V, 70, 141 ; XIX, 137.

Comitẻ de Leipzig. Guerre franco-allemande, III, 111.

Nouveaux statuts, XIX, 135.

\section{Variétés}

Société Albert en 1869 I, 70 1884 XVII, 65.

1871 IV, 44. 1885 XVIII, 28.

1883 XVI, $38 . \quad 1886$ XIX, 51.

Etablissements de Mme Simon, à Löschwitz, V, 141.

\section{Bibliographie}

Un train sanitaire pour Constantinople, par DE CRIEGERN, X, 55.

La guerre monténégrine, par FröLICH, X, 29.

Ia Croix-Rouge en Allemagne, par De CrIegers, XIV, 85.

\section{Wurtemberg}

\section{Croix-Rouge wurtembergeoise}

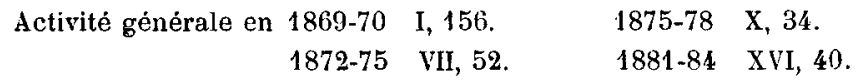

Guerre franco-allemande, II, 72, 228; III, 233; IV, 162.

Le Dr Hahn (nécrologie), XII, 26.

\section{Variétés}

Invalides et infirmières, $\mathrm{v}, 77$.

Eclairage des champs de bataille, XVII, 261.

\section{Bibliographie}

Les associations de dames, par HaHN, II, 160.

Les trains sanitaires, III, 49, 118.

Prisonniers de guerre français, par dE Chaulin, III, 50.

Manuel de chirurgie du Dr Landsberger, VI, 167.

Publication de la Sociétė (Mittheilungen), IV, 46. 
XIII

\section{AUTRICHE}

\section{Croix-Rouge autrichienne}

A) Socièté patriotique :

Activité générale en 1869 I, 172. $1878 \mathrm{X}, 82$.

1871 III, 186; IV, 7. 1879 XI, 108.

1872 V, $24 . \quad 1880$ XII, 126.

1873 V, $209 . \quad 1882$ XIV, 100.

1875 VlI, $132 . \quad 1883 \mathrm{XV}, 65$.

1876 VIII, $116 . \quad 1884$ XVI, 104.

Comité de Linz, I, 20.

Insurrection de Dalmatie, I, 80, 123.

Guerre franco-allemande, 11, 27.

Insurrection d'Herzégovine, VI, 175 ; VII, 1.

Les dix premières années de la Société, IX, 140.

Occupation de la Bosnie, IX, 285; X, 1, 9.

Projet de conférence internationale à Vienne en 1871, I, 125 ; II, 123;

IV, 145; - en 1883, X, 65; XII, 128; XIII, 32, 121.

B) Société de la Groix-Rouge:

Formation, XI, 46, 109.

Activité générale en 1880-81 XII, 125. 1885-86 XVII, 215.

1881-82 XIII, $126 . \quad 1886-87$ XVIII, 119.

1882-83 XIV, $97 . \quad 1887-88 \quad$ XIX, 99.

1883-84 XV, $138 . \quad 1888-89$ XX, 153.

1884-85 XVI, 102.

Insurrection de Bosnie, XIII, 193.

Abandon d'un projet de conférence internationale à Vienne, XIV, 68.

Le premier quart de siècle de la Croix-Rouge autrichienne, XVI, 10.

Guerre serbo-bulgare, XVII, 14, 21, 97.

Conférence internationale de Carlsruhe, XVIII, 57.

Franchise de port et facilités de transport, XVIII, 171.

\section{Variétés}

Conférence sanitaire à Vienne, V, 28, 94.

Exposition universelle de Vienne, IV, 8; V, 7, 91, 209.

Ordre teutonique, $\mathrm{V}, 25$.

Chevaliers de Malte, X, 82.

Confédération de vétérans, V, 159 ; XII, 195; XIV, 8, 101.

Inhumations en temps de guerre, XI, 48.

Soc. de la Croix-Blanche, XIV, 10.

L'électricité au service de la Croix-Rouge, XV, 31 . 
Traitement antiseptique, XVII, 223.

Emploi abusif de la Croix-Rouge, XVII, 282 ; XX. 44.

Correspondance des blessés en temps de guerre, XIX, 20.

Vingt-cinquième anniversaire de la Convention de Genève, XX, 179.

\section{Bibliographie}

Du transport des blessés, par Billnoth et Mundy, VI, 29.

Conférence sur la Convention de Genève, par Neumann, VI, 30.

L'humanité dans la guerre, par EсKеRT, VII, 77.

L'organisation sanitaire, d'après LaEw, VII, 132.

Le soin des malades, par le Dr GuRLT, XII, 196.

Le journal La Croix-Rouge, XVII, 28; XX., 180

Une brochure tyrolienne sur la Croix-Rouge, XVII, 104.

La Convention de Genève, par G. Roszkowsk, par le baron de NeumanN, XVIII, 58.

Carte statistique de la Croix-Rouge austro-hongroise, XVIII, 172.

\section{BELGIQUF}

\section{Croix-Rouge belge}

Personnel, VI, 33 ; XI, 14 ; XVI, 73.

A. Visschers (nécrologie), V, 222.

Le général Renard (nécrologie), X, 85 .

Le Dr Bougard (nécrologie), XV, 140.

Activité générale, $\mathrm{V}, 34$.

Guerre franco-allemande, II, 34 ; III, 67.

Projet de conférence internationale à Bruxelles, VJI, 87, 135, 182.

Reconnaissance officielle, XX, 47, 157.

Service de santé de la Croix-Rouge, XX, 90, 160.

\section{Variétés}

Soc. dite nationale belge de la Croix-Rouge, IX, 8, 226.

La Convention de Genève et la conférence diplomatique de Bruxelles, V, 197 ; VI, 9.

Congrès et exposition d'hygiène à Bruxelles, VI, 83, 186 ; VII, 137, 183, 189 ; VIII, 28 ; IX, 46.

Fondation de l'Institut de droit international, V, 99.

L'Institut de droit international et le Croissant rouge, IX, 142.

Voiture d'ambulance de Néef, I, 82.

Exposition de la Croix-Rouge à Anvers, XVI, 48, 105.

Concours de baraques à Anvers, XVI, 42, 44, 56, 97, 129.

Le grand concours de Bruxelles, XVIII, 174. 


\section{Bibliographie}

Rapport sur la. Conférence internationale de Berlin, par Visschers et Merchie, I, 23.

Journal d'une infirmière, par la Baronne de CrombrugGhe, III, 16.

Résections à Neuwied, par Hexfelder, IIi, 20.

La guerre et le droit international, par RoLin-JAEQUemyns, III, 73.

Souvenirs de la guerre franco-allemande, par VAN HoLsBeEk, IV, 10.

Secours aux blessés après la bataille de Sedan, par Merchie, VII, 194.

Assainissement des champs de bataille, par huBonN, VIII, 26.

Trois semaines aux ambulances, par OGER LAURENT, $\mathrm{X}, 144$.

Fédération des Soc. de secours, par Mornier. VIII, 12.

Une page de l'histoire de la Conrention de Genève, par Moynier, XVIII, 10.

Formation, XVI, 137.

BULGARIE

Statuts, XVII, 34.

Présidence, XIX, 69.

Guerre serbo-bulgare :

Appels, XVII, 11, 73.

Activitė, XVII, 105; XIX, $103 ; \mathrm{XX}, 97$.

\section{Variétés}

Essai d'une ouvre de secours, $\mathrm{X}, 86$

Adhésion à la Convention de Genève, XV, 142.

La Convention pendant la guerre serbo-bulgare, XVII, 225.

Le service sanitaire pendant la guerre serbo-bulgare, par le Dr Ror, XVIII, 63 .

\section{DANEMARK}

\section{Croix-Rouge danoise}

Reconstitution, VII, 14; VIlI, 28, 29.

Statuts, VIII, 58 ; XII, 198.

Premiers travaix, VIII, 52, 116.

Guerre turco-russe, IX, 47, 144.

Histoire rétrospective, VIII, 185.

Activité générale en 1876 VIII, $187 . \quad 1883 \quad \mathrm{XV}, 220$.

$\begin{array}{llrl}1877 & \text { IX, 234. } & 1884 & \text { XVI, } 153 . \\ 1878 & \text { X, 40. } & 1885 & \text { XVII, 284. } \\ 1879 & \text { XI, 154. } & 1876 \text { à } 1886 & \text { XVIII, 176. } \\ 1880 & \text { XII, 129. } & 1887 & \text { XIX, 156. } \\ 1881 & \text { XIII, 128. } & 1888 & \text { XX, } 183 . \\ 1882 & \text { XIV, 103. } & & \end{array}$




\section{Variétés}

La baraque dambulance de MM. Christoph et Unmack, par M. ADRIEN Peyrot, XVII, 38.

\section{FSPAGNE}

\section{Croix-Rouge espagnole}

Statuts, XX, 105.

Activité générale, I, 23, 173; II, 207; IV, 11; XVIJ, 109.

Guerre franco-allemande, II, 37.

Guerre carliste :

Activité générale, III, 196 ; IV, 14; V, 34; VI, 34, 188; VII, 16, 88.

Nuit du 11 décembre 1872, IV, 147.

La Croix-Rouge en Navarre, V, 161.

Appel aux neutres, V, 145, 194, 242; VI, 173.

Allocution de la duchesse de Medinaceli, VII, 140.

Le Comte de Ripalda (néorologie), VII, 139.

Décoration de la Croix-Rouge, XIV, 106.

Don Luis Perez Rico (nécrologie), XX, 50.

\section{Variétés}

La charité dans les guerres civiles, I, 175; V, 39.

Projet de M. MoxnIER, pour une institution judiciaire internationale, III, 203.

Adhésion de l'Espagne aux articles additionnels de la Convention de Genève.

IV, 11, 149.

Société de secours pour l'armée carliste, V, 104.

La trousse Landa pour le premier pansement, V, 223.

Les expériences de M. FurLey pendant la guerre carliste, VII, 17.

\section{Bibliographie}

Les Annales de la Croix-Rouge, par EnRIGH, VI, 37, 126.

Histoire des dames de la Croix-Rouge, X, 86.

La tactique sanitaire sur les champs de bataille, par le Dr LANDA, XI, 52.

\section{FRANCH}

\section{Croix-Rouge francaise}

Nom de la Société, XVIl, 49.

Personnel du Comité, I, 135; V, 170.

Le comte de Flavigny (nécrologie), v, 114.

Présidence, I, 178; XVII, 45, 233; XVIII, 17. 
Présidence d'honneur, IV, 85.

Décret du 2 mars 1878, IX, 240.

Décret du 3 juillet 1884, XV, 226.

Activité générale en: $\quad$ 1868-69, 1, 25.

1880 XII, 202.

1872 IV, 80,82;V,106. 1881 XIII, 93.

$1873 \mathrm{~V}, 42 . \quad 1882 \mathrm{XIV}, 108$.

1874 IV, $150 . \quad 1883 \mathrm{XV}, 146$.

1875 VII, 92. 1884 XVI, 109.

1876 VIII, 61. 1885 XVII, 226.

1877 IX, 236. 1886 XVIII, 181.

$1878 \mathrm{X}, 87.1887 \mathrm{XIX}, 111$.

$1879 \mathrm{XI}, 114 . \quad 1888 \mathrm{XX}, 162$.

Guerre franco-allemande :

Activité générale, II, 38; III, 24, 206.

Finances, V, 48.

Délégation de Bruxelles, II, 131.

Délégations régionales, II, 136 ; III, 146.

Lettre de Jules Favre, II, 212.

Ambulance de St-Cloud, III, 76.

Comités départementaux, III, 27.

Insurrection de Paris, II, 208; IV, 152.

Guerre turco-russe, IX, 57; X, 87.

Guerre serbo-bulgare, XVII, 112.

Expéditions d'Algérie et de Tunisie, XII, 205 ; XIII, 95.

Expéditions de Madagascar, du Tonkin et de l'Annam, XIV, 117; XV, 33;

XVII, 46, 47, 113; XVIII, 19.

Grandes: nanceuvres, XI, 15.

Mobilisation du 17e corps d'armée, XIX, 25.

Infirmeries de gares, XVIII, 17 ; XX, 52.

Exposition de Bordeaux, XIII, 143.

Exposition de Nice, $\mathrm{XV}, 92$.

Comités de dames, XII, 215 ; XIV, 112.

Commission de la lingerie, XVII, 46.

Conférence de délẻgués départementaux, V, 225 ; VI, 38, 86 ; VIII, $30 ; \mathrm{X}, 90$

XII, 16 ; XIII, 137 ; XVIII, 69.

Préparation du personnel sanitaire, $V, 50$.

Costume et insignes du personnel, X, 115.

Conférences instructives, XII, 57; XIII, 102; XVI, 74; XVII, 111.

Prix de Riencourt, I, 136.

Concours pour matériel sanitaire, IV, 14.

Comités départementaux:

Alger, XII, 134. 
Blois, XVII, 232.

Bordeaux, V, 53; VII, 196; VIII, 120; XI, 120; XII, 134; XIV, 114; XVIl, 290; XIX, 24, 163.

Grenoble, XIV, 149; XVI, 15; XVII, 113; XVIII, 76.

Havre, XVII, 290.

Lille, XIII, 100; XIV, 116; XVII, 289; XVIII, 77 ; XIX, 76.

Lyon, IV, 16.

Marseille, IV, 15; XII, 212 ; XVI, 12, $169 ;$ XX, 189.

Nancy, XII, 133; XIV, 115.

Orléans, XI, 120; XII, 135; XIV, 115; XVI, 169; XVII, 290; XIX, 162; $\mathrm{XX}, 188$.

Pau, VII, 94; XVIII, 127.

Strasbourg, III, 153.

\section{Variétés}

Association des Dames francaises, XII, 216; XIII, 209; XV, 94; XVlI, 117; XVIII, 21 ; XIX, 74.

Union des Femmes de France, XII, 135; XIII, 101, 209; XVII, 118; XVIII, 77.

Hospitaliers d'Afrique, IV, 17.

Bibliothèques militaires, IV, 156.

Conférences sanitaires à Paris, IX, 246, 291.

Comité protestant pour la guerre d'Orient, IX, 58, 289.

Société d'assistance aux mutilés paurres, X, 93; XIII, 146.

Lecture sur la Convention de Genève à l'Institut de France, I, 8.

Organisation des brancardiers militaires, XI, 18, 58.

Une rectification relative à la Convention de Genève, XIV, 153.

Constatation de l'identité des morts, XV, 153.

Un nouvel abus de la Croix-Rouge, XIX, 38.

Le musée international de Paris, par le Comte SÉrurIer, XVIII, 74.

L'improvisation du matériel des ambularıces, par le Comte de BEAUfort, XIII, 139.

Les ambulances flottantes, d'après le Dr RIANT, XIV, 71.

De l'évacuation des blessés et des ambulances de réserve, par le $\mathrm{Dr} H$. BernaRd, XV, 35.

Etude de M. PICHery sur les moyens de transport, XIV, 150.

Un appareil de transport pour les blessés, XI, 163.

Le brancard de M. Lónghà̇e, par le prof. D'Espine, XVIII, 191.

Sachets de pansement antiseptique, XIX, 164.

Transport des blessés en chemin de fer, XIX, 26.

Le train sanitaire de la Compagnie de l'Ouest, XVIII, 187 ; XIX, 28.

Congrès des cuvres d'assistance en temps de guerre, XX, 190. 


\section{Bibliographie}

Indications bibliographiques, I, 25 ; IV, 154.

La guerre et l'humanité, par DE CAzenove, I, 88.

Mission des femmes en temps de guerre, par Mme Monod, 1I, 141.

Etude sur la Convention de Genève, par G. Moynier, III, 9.

Rapport de M. Piotrowski (guerre franco-allemande), IlI, 35.

Chirurgie militaire, par L. LE FORT, III, 91; IV, 19.

Construction des ambulances temporaires, par DEMoGer, III, 89.

Ambulance de la Còte d'Or, par DUGAst, III, 149.

Histoire du blocus de Metz, par Greiclors, V, 54.

La Convention de Genève, d'après La Guéronnière, VII, 23.

Casernes et camps permanents, par Marvaud, V, 58.

Les invalides en France, par DE RIEvcourt, VII, 27, 102.

Transport des blessés chez les anciens, par PÉTrequin, VII, 28.

Questions philanthropiques, par DE BEAUFORT, VII, 95.

Manuel de la dame de charité, par le Dr Chenu, VII, 198.

Manuel de droit international pour les écoles militaires, IX, 53.

Annuaires et Bulletins de la Croix-Rouge française, X, 14; XII, 215 ; XVII, 110.

Guide médical de l'officier détaché, par le Dr Marmonier, XI, 121.

Appareil de transport, par DE BEAUFORT, XII, 219.

L'assistance aux blessés, d'après le Dr Bouloumí, XllI, 147.

La Convention de Genève et les ambulances internationales, d'après M. ALIX, XIII, 213

La Croix-Rouge à l'Exposition universelle de 1878, d'après le Dr Gruby, $\mathrm{XI}, 57 ; \mathrm{XV}, 97,150$.

Un commentaire sur la Convention de Genève, par J. Guelle, XV, 151.

Manuel du brancardier, du Dr F. Gross, par le Dr APPIA, XV, 232.

Transport par chemin de fer des blesșès et des malades militaires, d'après le Dr Redard, par le Dr Appia, XVI, 170.

Traité de chirurgie de guerre, par E. Delorme, XIX, 71.

Album du matériel d'ambulance de la Croix-Rouge française, XIX, 160.

\section{GRANDE-BRETAGNE}

\section{Croix-Rouge anglaise}

Comité provisoire, I, 27.

Questionnaire aux agents, III, 36.

Règle pour les agents, VIII, 70.

Exposition universelle de Vienne, V1, 42.

Instruction d'infirmières, XII, 220; XVII, 120. 
Considérations générales, XVII, 49.

Critiques, XVII, 132.

Guerre du Zoulouland, X, 47, $117 ;$ XVII, 120.

"de la Basse-Egypte, XIV, 14, $120 ; \mathrm{XVl}, 75$.

" du Transvaal, XVII, 120.

"du Soudan égyptien, XV, 237; XVl, 75; XVIL, 120, 127.

- franco-allemande, II, 42 ; III, 36, 93.

"turco-serbe, VII, 200 ; VIII, 34, 69, 121 ; XI, 25.

" russo-turque, VIII, 125; IX, 59, 145, 248 ; X, 17; XI, 25.

» serbo-bulgare, XVII, 236.

\section{Variétés}

Ordre de St-Jean de Jérusalem, VI, 40; VII, 143, 200; X, 47 ; XI, 22; XII, 20 ;

XVI, $18 ; \mathrm{XIX}, 166$.

Ambulance de l'Ordre de St-Jean, IX, 154, 248, 303; X, 46, 123 ; XII, 20 ;

XIII, 45; XIV, 154; XVI, 19, 173 ; XVIL, 291.

Abus du brassard, V, 116.

Ordre du Temple, VI, 189.

Instruction de brancardiers, VIII, 72.

Philanthropie pendant la guerre, VIII, 73.

Le Comité de Stafford House pendant la guerre turco-russe, X, 121.

Les dernières guerres, XI, 21.

J.-H. Porter (nécrologie), XI, 58.

Congrès médical à Londres, XII, 136, 224.

Les femmes de soldats dans les ambulances, XIII, 50.

Les événements d'Egypte et la Convention de Genève, XIV, 22.

Décoration royale de la Croix-Rouge, XIV, 118.

La Croix-Rouge à l'Exposition d'hygiène de Londres, XV, 99, 234.

\section{Bibliographie}

Le transport des blessés, par le Dr Longmore, I, 28.

La guerre et la charité, par G. Moynier et APPIA (traduit par FurLey), I, 181.

Deux écrits du Dr LongmoRe, II, 214.

Efforts d'un volontaire, par FurLey, IV, 88.

Manuel de chirurgie, par Ponter, VI, 190.

Conférence de FunLEY, VH, 145.

Manuel du Dr Moffit, VII, 202.

Exposition d'hygiène de Bruxelles, par le Dr LONGMORE, VIII, 78.

Plaies par armes à feu, par le Dr LongMone, IX, 297.

Un nouveau manuel pour les lois de la guerre, XI, 163; XII, 23, 29.

Manuel de législation militaire, XV, 236. 
Un discours du professeur LONGMORE, XVI, 16.

L'histoire de la Croix-Rouge, par G. Moynien, XIV, 77.

Les armées françaises et anglaises en Crimée, d'après le Dr Longmone, $\mathrm{XIV}, 125$.

\section{GRÈCE}

\section{Croix-Rouge grecque}

Formation, VIII, 176, 195; IX, 66.

Personnel du Comite central, XII, 58.

Activité générale en : $1878 \mathrm{X}, 94 . \quad 1884 \mathrm{XVI}, 114$.

$1879 \mathrm{XI}, 28,123 . \quad 1885$ XVII, 137.

1880 XII, 59. 1886 XVIII, 128.

1881 XIII, $149 . \quad 1887$ XIX, 76.

$1882 \mathrm{XV}, 42 . \quad 1888 \mathrm{XX}, 200$.

$1883 \mathrm{XV}, 101$.

Conflit gréco-turc, IX, 113, 155.

Réfugiés de Turquie, IX, 252; X, 23; XI, 59.

Blessés de Thessalie à Athènes, XI, 61 .

Tremblement de terre de Ghio, XII, 137, 226.

Ambulance de la Société, XX, 55.

\section{HONGRIE}

Croix-Rouge hongroise

Formation, XIII, 13.

Statuts, XIII, 53.

Activité générale en: 1881 XIII, $217 . \quad 1886$ XVIII, 134.

1882 XIV, $126 . \quad 1887$ XIX, 117.

1884 XVI, $176 . \quad 1888 \mathrm{XX}, 204$.

1885 XVII, 292.

L'hôpital Elisabeth, XIII, 56 ; XVI, 178.

Guerre serbo-bulgare, XVII, 143.

Diffusion de la Convention de Genève dans l'armèe, XIX, 120.

Répression de l'usage abusif de la Croix-Rouge, XX, 201.

\section{Bibliographie}

Publications de la Croix-Rouge hongroise, XIII, 152.

Carte statistique de la Croix-Rouge austro-hongroise, XVIII, 172.

\section{ITALIE}

\section{Croix-Rouge italienne}

Statuts, VI, 193.

Règlement organique, XIX, 168. 
Règlement pour le temps de guerre, XVII, 74, XIX, 171.

Personnel du Comité central, XI, 29; XVI, 78; XVIII, 23.

Les Dr Castiglioni et Barbieri (nécrologie), III, 101.

Le Dr Trezzi (nécrologie), VI, 199.

Transfert du Comité central à Rome, VI, 148, 191; VII, 148.

Activité générale en: 1868 I, $31 . \quad 1881_{1} 1885$ XVII, 297.

1869 I, 182. 1886 XVIII, 140.

1877 VIII, $200 . \quad \$ 887$ XIX, 123.

1881 XIII, 154. 1888 XX, 171.

1885 XVI, 117; XVII, 300.

Guerre franco-allemande, II, 50.

Guerre turco-russe, IX, 308.

Comitè de Padoue de 1868 à 1870, III, 155.

Comité de Milan en 1879, XI, 124.

Enquête sur les ressources nationales, IX, 67.

Ia Croix-Rouge et l'ordre de St-Jean, VIII, 202; IX, 306.

Grandes manœuvres de l'armée, XI, 169; XII, 65.

Rapport au roi, XII, 140, 231.

Tremblement de terre d'Ischia, XIV, 157; XV, 155.

Exposition de Turin, XV, 238.

Emprunt à lots, XVII, 57.

Trains hôpitaux, XVI, 21 ; XVIII, 193.

Union des dames de la Croix-Rouge, XIX, 130.

Réunion des présidents des sous-comitès, XX, 174.

\section{Variétés}

Exposition maritime à Naples, I, 139.

Lettre du Dr Palasciano sur la Convention de Genève, III, 103.

La législation militaire et les médecins, V, 63

Monument de la Croix-Rouge, XIV, 32.

L'article sept de la Convention de Genève, par Maggronani, XVI, 25.

\section{Bibliographie}

Annales de médecine de Griffini, V, 125.

Dictionnaire de l'infirmier, par TORELLI, VII, 203.

Véhicules pour blessés, par LoCATI, VIII, 127.

La Convention de Genève, par OLrvi, X, 101, 125.

Manuel de chirurgie militaire, par le Dr Moretrini, XV, 158.

Etude sur la Convention de Genève par Bosco, par M. Basso, XV, 242.

Les porteurs de blessés, par le Dr OGNIBENe, XVI, 119.

Bulletin de la Croix-Rouge italienne, X, 101 ; XVII, 296; XVIII, 192.

Le journal " La Croix-Rouge ", XVIII, 148. 


\section{LUXFMBOURG}

\section{Croix-Rouge luxembourgeoise}

Guerre franco-allemande, II, 53.

Guerre turco-russe, IX, 157.

Le Dr Pierre Schmit (nécrologie), X, 127.

Dissolution de la Société, XIV, 78.

\section{Variétés}

Le corps médical luxembourgeois en 1870-71, III, 156, 217.

Adhésion à la Convention de Genève, XIX, 173.

\section{MONTENEGRO}

Formation, VlI, 66.

Croix-Rouge monténégrine

Statuts, VII, 65.

Mission du Comité international, VII, 55.

Appels, VII, 68; VIII, 79.

Guerre contre les Tures, VII, 210; IX, 69.

Reconstitution de la Société, XVII, 160.

Histoire de la Société, XIX, 175.

\section{Variètés}

La guerre contre les Turcs, VIII, 34 ; IX, 62.

Adhésion à la Convention de Genève, VII, 5 .

\section{NORWEGE}

Croix-Rouge norwégienne

Présidence du Comité central, XII, 73.

Guerre franco-allemande, II, 57.

Activité générale en 1887-88, XX, 118.

\section{Bibliographie}

Chariot pour le transport des blessés, par SMITH, XI, 124.

\section{PAYS-BAS}

Croix-Rouge néerlandaise

Personnel du Comité central, III, 218; VI, 148; XIII, 58, 103.

Le Dr Basting (nécrologie), II, 144.

L'amiral van Karnebeek (nécrologie), Ill, 38. 
M. J. Bosscha (nécrologie), VI, 47.

Le Dr Verwey (nécrologie), VI, 202.

Le général de Stuers (nécrologie), XIII, 69.

Activité générale en : 1867-69 I, 31. $1881-82 \quad \mathrm{XV}, 109$.

1874-72 V, $229 . \quad 1885-87 \quad$ XIX, 77.

1878-79 XI, 61 .

Guerre franco-allemande, II, 58, $214 ;$ III, 218.

Guerre d'Atchin et Comité de Batavia, V, 64, 126, 232 ; VI, 46, 149; VII, 211 IX, 158.

Guerre turco-russe, VIII, 128, 205; IX, 71, 257; X, 24.

Guerre du Transvaal, XII, 74, 142, 233; XIII, 158, 160.

Comités sectionnaires. I, 93; VI, 45; XI, 127 ; XII 144.

Division du travail, VII, 30,35 .

Exposition de La Haye, I, 7, 35, 140.

Concours sur les mesures à prendre en cas de guerre, I, 93.

Exposition d'Amsterdam, XIV, 164.

Instruction des infirmiers et des infirmières, XVII, 57.

La question des décorations, XVIII, 79.

\section{Variètés}

Batterie de cuisine portative du colonel van TuylL, I, 94.

Médaille de la Croix-Rouge, II, 146.

Expériences de la guerre franco-allemande, IV, 26.

Abus du brassard, V, 66.

La Convention de Genève aux Indes, V, 172, 234.

Le léchophore de Moors, V, 174; VI, 50.

Brancard du Dr Becking, Vl, 150.

Embarquement des blessés, IX, 255.

Société pour procurer des membres artificiels, XIII, 219; XIV, 83.

Effet des balles de fusil, XVII, 163.

\section{Bibliographie}

Des moyens de transport, par van Dommelen, 1, 183.

La Croix-Reuge sur mer, par Ferguson, IV, 27.

L'Exposition de Vienne, par GoRI, V, 238.

Bandages dits "transports-rotang ", par le Dr de MooIJ, XII, 145.

La flore des Indes appliquée au soin des blessés, d'après le Dr DE Moou, XIV, 79.

Transport des bléssés par voies ferrées, par le Dr GoRI, XIV, 161. 
XXV

\section{PORTUGAL}

\section{Croix-Rouge portugaise}

Formation, I, 151.

Réorganisation, II, 147.

Guerre franco-allemande, II, 66; III, 158.

Reconstitution, XVIII, 80,153; XIX, 40.

Statuts, XIX, 41.

Variètés

Abus du signe de la Croix-Rouge, XIX, 134,

\section{Bibliographie}

Bulletin de la Croix-Rouge portugaise, XIX, 133.

\section{ROUMANIF}

Croix-Rouge roumaine

Formation, VII, 159.

Statuts, VII, 212.

Activité générale; X, 129 ; XIV, 130.

Guerre turco-serbe, VII, 216; VIII, 206.

Guerre turco-russe, IX, 81, 165.

Guerre serbo-bulgare, XVII, 164.

\section{Varietés}

Adhésion à la Convention de Genève, VI, 14, 65.

\section{RUSSIE}

\section{Croix-Rouge russe}

Personnel du Comité central, V, 1;8; XV, 114.

Le Dr Hubbenet, (nécrologie), V, 187.

Le général Baumgarten (nécrologie), XIV, 130.

Nom de la Socièté, X. 48.

Règlement de guerre, XII, 238.

Activité génêrale en : 1868 I, 44, 49. $1879 \quad \mathrm{X}, 135 ; \mathrm{XI}, 30,176$.

$1872 \quad \mathrm{~V}, 179 . \quad 1881 \quad \mathrm{XIII}, 63$.

1873 VI, 53, 97. 1883 XV, 114; XVI, 83, 125.

$1874, V, 165 . \quad 1884 \quad \mathrm{XV}, 160$.

1875 VII, $218 . \quad 1885$ XVII, 304,

$1878 \mathrm{X}, 49,102$ 
Exposition de St-Pétersbourg, I, 187.

Guerre franco-allemande, II, 66; III. 226.

Expédition de Khiva, V, 181.

Incendie de Morschansk, VI, 212.

Insurrection de l'Herzégovine, VII, 105.

Guerre turco-serbe, VII, 233.

Guerre turco-russe, VIII, 210; IX, 87, 167, 258; X, 49, 51; XII, 188; XIV, 35.

Epidémie d'Astrakan, X, 52.

Incendie d'Orenbourg, $\mathrm{X}, 104$.

Expédition des Akhals-Tékés, XII, 84, 152.

Quatorzième anniversaire de la Société, XII, 150.

La Société en temps de paix, XII, 241.

Ouverture d'un concours permanent, XIV, 83.

Cures de bains pour les officiers, XIV, 132.

La Croix-Rouge sur les côtes du Mourman, XV, 251.

Guerre serbo-bulgare, XVII, 164, 251 ; XVIII, 203.

Institutions de la Société, XIX, 78.

Comitė d'Odessa, XV, 163, 248; XVIII, 85.

) de Moscou, XVI, 29.

n de Varsovie, XVI, 27; XVIII, 84.

n d'Arkhangel, XVIII, 25.

\section{Variétés}

Assistance des blessés en Russie, IV, 158.

Soeurs de l'exaltation de la Croix, I, 50, 97.

Sours de la charité de St-George, IV, 43.

Lazaret évangélique de Sistowa, IX, 264, 313.

Baraque-lazaret à St-Pétersbourg, III, 168.

Association française de bienfaisance à St-Pétersbourg, III, 160.

Transport des malades et des blessés, I, 185; VI, 208.

Flotille du Danube, par Recke, VIII, 131.

Diagnostic et extraction des projectiles par MiLLIot, II, 148.

Application de la Convention de Genève à la marine, $\mathrm{XI}, 33$.

La Société finlandaise et l'ambulance finnoise, XI, 38.

Le capital de Sébastopol, XII, 88.

Les enseignements de la guerre turco-russe, par le Cie Prozor XI, 63 ; XII, 89, 154.

Les évacuations par chemin de fer, par le Cle Prozor, XIII, 104.

La guerre turco-russe et la Convention de Genève, d'après le professeur de Martens, XIV, 36.

Décoration de la Croix-Rouge, XIV, 177.

Exercices de porteurs de blessés, XVI, 26. 


\section{XXVII}

Le couvent de l'Epiphanie à Kostroma, XVI, 86.

Communauté des sœurs de St- George, XVII, 63; XVIII, 27.

\section{Bibliographie}

Hôpitaux russes en Crimée, par Hubbenet, III, 161.

Baraques-lazarets, par le Dr Berthenson, III, 166; VI, 101.

Hòpitaux français et allemands, par le 1)r Pirogoff, III, 111, 162.

Service sanitaire à Plevna, par le Dr Koccher, XI, 130.

Etat sanitaire des troupes russes et leurs pertes dans la guerre turco-russe, par le Dr KGeHer, XIII, 112

Etude sur la Convention de Genève, par Ch. Iwanowski, par DE Martens, $\mathrm{XV}, 252$.

Fourgons et charrettes pour le transport des blessés, par TAHDENT, XVI, 31.

\section{SERBIE}

\section{Croix-Rouge serbe}

Fondation, VII, 118, 151.

Statuts, VII, 153; IX, 179.

Personnel du Comité central, XV, 166; XVI, 81; XVII, 254; XIX, 81

Activitẻ générale en : 1879-80 XII, 170. 1885-86 XVIII, 215.

1880-81 XV, $166.1886-87$ XIX, 51.

1881-83 XVII, $66 . \quad 1887-88 \quad \mathrm{XX}, 133$.

1884 XVII, 307.

Guerre turco-serbe, VII, 120, 235; IX, 8, 95; X, 138.

Maison de la Croix-Ronge à Belgrade, $\mathrm{X}, 145$.

Guerre serbo-bulgare, XVII, 12, 170

I)ixième anniversaire de la Société XVIII, 86.

\section{Variétés}

Adhésion à la Convention de Genève, VII, 117.

Violations de la Convention de Genève, VII, 237.

Instruction sur la Convention de Genève, IX, 101, 176.

Prisonniers serbes en Turquie, IX, 266.

\section{Bibliographie}

Album de la Croix-Rouge, VlII, 133.

Conférences du Dr VladaN GeoRGewitch, VII, 150. 


\section{XXVIII}

\section{SUÈDE}

Croix-Rouge suédoise

Personnel du Comité central, IK, 94 ; V, 71; VII, 51.

Activité générale en:

\begin{tabular}{llll}
1869 & I, 99. & 1880 & XII, 114. \\
1870 & II, 153. & 1881 & XII, 75. \\
1871 & III, 169. & 1882 & XIV, 134. \\
1872 & IV, 94. & 1883 & XV, 170. \\
1874 & VI, 102. & 1884 & XVI, 87. \\
1875 & VII, 108. & 1885 & XVII, 255. \\
1876 & VIII, 134. & 1886 & XVIII, 150. \\
1877 & IX, 183. & 1887 & XIX, 138. \\
1878 & X, 58. & 1888 & XX, 135. \\
1879 & XI, 34. & & \\
\hline
\end{tabular}

Guerre franco-allemande, II, 69, 159, 220.

Histoire générale de la Société, VII, 239.

Le colonel Staaff (nécrologie), XIX, 54.

\section{Variétés}

Statuts de la Société samaritaine, XV, 175.

\section{Bibliographie}

Exposition et conférence de Paris, par le Dr GräHs, I, 156.

Exposition de Vienne, par le Dr GräHs, VI, 214.

\section{SUISSE}

\section{Croix-Rouge suisse}

Personnel du Comité central, III, 232.

Statuts, XVIII, 89.

Activité générale en : 1871 III, 41.

1884 à 1886 XVIII, 30.

$1883 \mathrm{XV}, 118$. 1887 $\mathrm{XIX}, 184$.

1884 XV, 176; XVI, 41. 1886 à 1888 XX, 209. 1886 XVIII, 89.

Section de Zurich, I, 51.

Guerre frąco-allemande, II, 70.

Réorganisation, XII, 220.

\section{Variétés}

Enseignement de l'hygiène aux soldats, l, 53.

Perfectionnement du matériel officiel, I, 55, 102; 1lI, 48.

Règlement pour le transport des blessés, I, 100.

Transport des blessés en chemins de fer, I, 104; VI, 58. 
Ambulance Pranco-suisse, II, 223.

Institut de Bâle pour membres artificiels, III, 114.

Comité de Bâle pour les prisonniers de guerre, II, 92.

Conférences de médecins militaires, III, 171.

Stations de convalescents, IV, 45 .

Malades français internés en Suisse, IV, 159; V, 189.

Port du brassard en temps de paix, VI, 59.

Barbe Schinner, par DAgueT, VI, 61.

Opinion d'un médecin sur la Convention de Genève, VII, 157.

L'Institut de droit international et la guerre d'Orient, VIII, 217.

Machine à glace pour les ambulances, $\mathrm{X}, 30$.

Congrès d'hygiène à Genève, XIII, 182.

Sœurs de la Croix-Rouge, XIV, 89.

Exposition de Zurich, XIV, 139.

Organisation du service de santé officiel, XIV, 178.

Société des Samaritains à Berne, par ERNest MöckLy, XVII, 70.

Le Conseil fédéral et la Convention de Genève, XVII, 179.

Les sociétés de secours aux invalides, par A. DoRET, XVIII, 37.

\section{Bibliographie}

Les Sociétés de secours en temps de paix, par HrLty, I, 52.

Essai sur la médecine militaire, par Weinmans, I, 188.

Activité d'un lazaret prussien, par BuRGKaRdT, III, 43.

La Société Winkelried, par Escher, V, 72.

Discours du Dr ApPIA au Congrès de Bruxelles, VIII, 79.

La Croix-Rouge: son passé et son avenir, par G. MoynIER, XIII, 226.

De l'improvisation des moyens de traitement, d'après lè Dr CuBACH, par le Dr Ferrik̀re, XV, 177.

Le service sanitaire dans les montagnes, d'après le Dr Frölrah, XVIII, 93. Aménagement des voitures de guerre pour le transport des blessés, d'après le Dr Frölich, par le Dr ApPIA, XIX, 82.

\section{TURQUIE}

\section{Croissant rouge ottoman}

Formation, I, 57.

Dr Abdullah Bey (nécrologie), VI, 63.

Reconstitution, VII, 161; VIII, 39, 80.

Règlement, VIII, 80.

Guerre turco-serbe, VII, 120.

Guerre turco-russe, VIII, 140, 222; IX, 102, 189, 268, 314; X, 31.

Réponse à l'Institut de droit international, IX, 108, 142.

Réorganisation, XVII, 82.

Un incident admiıistratif au sein de la Société ottomane, XX, 211. 


\section{ASIE}

\section{CHINE}

Variétés

De l'introduction de la Croix-Rouge en Chine, par le Dr Mantin, XIII, 37. Manuel des lois de la guerre, XIV, 147.

\section{INDE}

\section{Variétés}

Les lois de la guerre chez les anciens Hindous, par Paul Oltramare, XII, 61.

\section{JAPON}

\section{Croix-Rouge japonaise}

Formation, XVIII, 157.

Statuts, XVIII, 194.

Vingt-cinquième anniversaire de la fondation de la Groix-Rouge, XX, 112. Assemblée générale, XX, 175.

\section{Variétés}

L'ambulance japonaise et le Comité international, V, 11.

La Convention de Genève au Japon, VIII, 1.

Société Haku-ai-sha, XV, 104.

Adhésion à la Convention de Genève, VIII, 1 ; XVII, 244.

\section{PERST}

Variétés

Adhésion à la Convention de Genève, V, 16; VI, 67.

\section{AFRIQUE}

\section{CONGO}

Croix-Rouge congolaise et africaine

Formation, $\mathrm{XX}, 76$.

Statuts, XX, 101, 182.

Premiers travaux, XX, 182. 
Variètés

Adhésion à la Convention de Genève, XX, 100.

\section{RÉGIONS DIVFRSES}

Nouvelles diverses, XI, 5, 103; XII, 9, 10; XIII, 86.

\section{AMÉRIQUE}

\section{REPUBLIQUE ARGENTINE}

Croix-Rouge argentine

Formation et origines, XIII, 10, 193.

Statuts, XIII, 19.

Activité générale en 1881-82, XIII, 202.

Formation d'une école de Samaritains, XVI, 65.

Variétés

Adhésion à la Convention de Genève, XI, 13.

\section{BOLIVIF}

Variètés

Adhésion à la Convention de Genève, XI, 12.

\section{BRÉSIL}

Variétés

Le Brésil et la Convention de Genève, XVIII, 124.

\section{CHIUI}

\section{Variétés}

Guerre contre le Pérou et la Bolivie, X, 81, 110.

Adhésion à la Convention de Genève, $\mathrm{X}, 111 ; \mathrm{XI}, 13$.

\section{Bibliographie}

Ce que c'est que la Croix-Rouge, par Edouard Sève, XI, 106. 
XXXII

\section{ÉTATS-UNIS}

\section{Croix-Rouge américaine}

Premiers travaux, I, 24.

Guerre franco-allemande, II, 38.

Dissolution, VII, 159.

Reconstitution, XIII, 185.

Statuts, XIII, 205.

Subvention de l'Etat, XIII, 208.

Activitè générale, XV, 89; XVI, 107; XVIII, 126; XIX, 70.

Inondations de l'Ohio et du Mississipi, XV, 143.

La Croix-Rouge aux grandes manceuvres, XVIII, 179.

Inondations en Pennsylvanie, XX, 161 .

\section{Variétés}

Miss Barton, VIl, 90.

Adhésion à la Convention de Genève, XIII, 41, 92, 133.

Des conditions essentielles que doivent remplir les secours volontairea, par Ch. SMart, XVI, 157.

\section{Bibliographie}

Excisions de la tête du fẻmur, par OTIS, I, 86.

Baraques et hôpitaux militaires, III, 22.

Cas de chirurgie observés de 1865 à 1871, III, 144 .

Histoire médicale et chirurgicale de la guerre de sécession, V, 164; VHI, $30 ; \mathrm{XI}, 55$.

L'ambulance américaine à Paris en 1870-71, par Evans, V, 167.

Hygiène de l'armée américaine, VII, 21.

Transport des blessés, par OTIs, VIII, 193.

Un nouveau journal de la Croix-Rouge, XV, 87.

Catalogue de la bibliothèque de l'administration sanitaire, XVII, 44.

Notice sur la Croix-Rouge, par SHecdon, XVIII, 14.

\section{PEROU}

\section{Croix-Rouge péruvienne}

Formation, XI, 98.

Personnel du Comité central, XI, 171 ; XIII, 103.

Le Dr Odriozola ((nécrologie), XIX, 183.

Appel, XII, 5. 
XXXIII

Ambulance du Callao, XII, 80.

Histoire rétrospective, XIII, 164

Reconstitution, XVII, 248.

Activité générale, XIII, 60; XVIII, 149; XIX, 180.

\section{Variètes}

Traité entre le Pérou et la Bolivie, V, 84.

Adhésion à la Convertion de Genève, X, 112; XI, 128.

Application de la Convention de Genève, XI, 172 ; XII, 23.

\section{SAN SALVADOR}

Varietes

Adhésion à la Convention de Genève, VI, 110. 


\section{INDEX}

\section{DE LA TABLE DES VINGT PREMIERS VOLUMES}

\begin{tabular}{|c|c|c|c|c|c|c|c|c|c|c|c|c|c|}
\hline \multicolumn{13}{|c|}{ Pages } & Pages \\
\hline interna & atio & nal & & & . & & 3 & Pays-Bas . . . & & & . & & 23 \\
\hline & $E u$ & rox & pe. & & & & & Portugal . . . & . . & 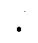 & . & & 25 \\
\hline Allemagne & . & - & - & & 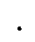 & & 7 & Roumanie. . . & . . & . & . & - & 25 \\
\hline Généralités & . & & • & & . & & . & Russie . . . . . & . . & . & . & & 25 \\
\hline Prusse . & . & & . & & . & & . & Serbie . . . . & . . & . & . & - & 27 \\
\hline Pays connex & xes & & & & . & & . & Suède . . . . . & . & . & - & - & 28 \\
\hline $\mathrm{Bad}$ & . & & - . & - & $\cdot$ & & . 10 & Suisse . . . . . & . . & . & - & . & 28 \\
\hline re. & . & . . & . & . & 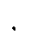 & & . 11 & quie. . . . . & . & . & . & . & 29 \\
\hline Hesse . . & . & . . & . . & . & . & & . 11 & Asic & & & & & \\
\hline Saxe. . & - & - . & . . & - & - & & . 12 & $\cdot \cdot \cdot$ & . & & . & . & 30 \\
\hline Wurtemberg & & . . & . . & & . & & . 12 & Inde . . . . & - & & . & 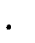 & 30 \\
\hline Autriche & . & . . & . . & & . & & . 13 & Japon. . . . & . & & - & . & 30 \\
\hline Belgique . . & . & . . & . . & & . & & - 14 & Perse . . . & & & - & - & 30 \\
\hline Bulgarie . & . & . . & . & & . & & 1 & Afriq & & & & & \\
\hline Danemark . & . & & & & - & & . 15 & Congo . . . . & . . & & . & . & 30 \\
\hline Espagne . . & - & . . & • & & . & & - 16 & Régions diverses. & . . & & 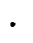 & . & 31 \\
\hline France & 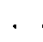 & & & & * & & . 16 & Améri & ique & & & & $=$ \\
\hline e-Bretag & gne & & & & . & & . 19 & lique Argenti & ine. & & . & 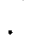 & 31 \\
\hline Grèce . . . & • & & & & " & & - 21 & Bolivie . . . & . & & . & . & .31 \\
\hline Hongrie. . . & . & & & & • & & - 21 & Brésil . . . & . & & 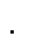 & . & 31 \\
\hline Italie. . . . & . & & & & - & & 21 & Chili . . . & • & & - & - & - 31 \\
\hline Luxembourg. & ${ }^{\circ}$ & & & & & & 23 & Etats-Unis. . . & & & & & 32 \\
\hline Montenegro. & ${ }^{\circ}$ & & & & & & . 23 & Pérou & & & & ${ }^{\circ}$ & . 32 \\
\hline Norwège & - & & & & & & . 23 & San-Salvador & & & & - & 38 \\
\hline
\end{tabular}




\section{ADRESSES DES COMITÉS CENTRAUX}

(SUITE)

(Voir la deuxième page de cette couverture.)

GRÈCE. - M. Marc Renieris, gouverneur de la Banque de Grèce, président de la Société grecque de secours aux blessés, à Athènes.

HESSE. - M. A. Weber, président du Ministère des Finances, etc., pour la Société de secours aux militaires blessés, à Darmstadt.

HONGRIE. - Direction de la Société hongroise de la CroixRouge, Kettenbrückengasse, 1, à Budapest II.

ITALIE - Présidence de la Croix-Rouge italienne, à Rome.

JAPON. - Société japonaise de la Croix-Rouge, à Tokio.

MONTÉNÉGRO. - Mgr. Mitrofan, archevêque métropolitain, président de la Société monténégrine de la Croix-Rouge, à Cettigné.

NORWÉGE. - M. le capitaine Peter Berg, secrétaire du Comité central norwégien de secours aux militaires blessés, à Christiania.

PAYS-BAS - M. le $D^{r} B$. Carsten, secrétaire-général, pour le Comité central de la Société néerlandaise de la Croix-Rouge, à La Haye.

PÉROU. - M. Ch. SotomaYor, secrétaire perpétuel de la Société péruvienne de la Croix-Rouge, à Lima.

PORTUGAL. - Société portugaise de la Croix-Rouge, à Lisbonne.

PRUSSE - Comité central de l'association prussienne de secours aux militaires blessés et malades. Wilhelmstrasse, 73, à Berlin.

ROUManie. - S. Exc. le Prince Démétre Ghika, président, pour la Société roumaine de la Croix-Rouge, à Bucharest.

RUSSIE. - Comité central russe de la Groix-Rouge. Rue des Ingénieurs, 9, à St-Pétersbourg.

SAXE. - M. Fr. de Criegren-Thumitz, président, conseiller intime de gouvernement de S. M. le roi de Saxe, à Bautzen (Saxe royale).

SERBIE. - M. le général M. Lechüanine, président de la Sociétẻ serbe de la Croix-Rouge, à Belgrade.

SUĖ̉DE. - Comité central de la Société suédoise de la CroixRouge (Svenska Föreningen Röda Korset), à Stockholm.

SUISSE. - M. le pasteur Wennly, secrétaire de la Société suisse de la Croix-Rouge, à Aarau.

TURQUIEx - Société ottomane de secours aux militaires blessés, Hòtel de l'administration sanitaire centrale de l'Empire, à Galata, Constantinople.

WURTEMBERG. - Société sanitaire, à Stuttgart. 


\section{SOMMAIRE}

Comité international. - Concours sur l'emploi abusif du signe et du nom de la Croix-Rouge, 177. - Ouvrages reçus et publications nouvelles, 178.

Autriche. - Vingt-cinquième anniversaire de la Convention de Genève, 179.

- Le journal Das rothe Kreuz, 180.

Bade. - La Société badoise des dames en 1888, 181.

Congo. - Premiers travaux de la Société congolaise et africaine, 182. Revision des statuts de la Société congolaise, 182.

Danemark. - La Société danoise en 1888, 183.

France. - Comité du Loiret, 188. — Comité des Bouches-du-R hône, 189. -

Congrès des ceuvres d'assistance en temps de guerre, 190.

Grèce. - La Sociẻté hellénique en 1888, 200.

Hongrie. - Répression de l'usage abusif de la Groix-Rouge, 201. - La Société hongroise en 1888, 204 .

Prugse. - La Société patriotique des dames, 208.

Suisse. - La Société suisse de 1886 à 1888, 209.

Turquie. - Un incident administratif au sein de la Société ottomane, 210.

Table des Matières de la XXe année, 214.

Table générale des matlères.des vingt premières années.

Le Bulletin international paraît régulièrement tous les trois mois (vers la fin des mois de Janvier, Avril, Juillet et Octobre).

Prix, franco, pour un an et pour tous les pays de l'Union postale six franes. - Prix du numéro Fr. 1,50.

On s'abonne auprès du Comité international ou des divers Comités centraux (voir leurs adresses ci-contre). 